\title{
Jahrestagung 2003
}

Für ihre Jahrestagung 2003 war die Vereinigung der Deutschen Staatsrechtslehrer in der Freien und Hansestadt Hamburg zu Gast und konnte insbesondere bei dem Empfang im Hamburger Rathaus - etwas von der Weltläufigkeit dieser historischen Handelsmetropole spüren. Dass Hamburg nur sog. Schmuddelwetter zu bieten hätte, wurde jedenfalls für die ersten zwei Tage eindrucksvoll widerlegt: So konnten sich Alster, Hafen und Speicherstadt im besten Lichte zeigen. Für die gute Organisation dieser gelungenen Tagung geht unser Dank auch an dieser Stelle an die Hamburger Kollegen, vor allem an Herrn Stefan Oeter und sein aufopferungsvolles Team.

In der traditionell am Mittwoch stattfindenden Mitgliederversammlung gedachte die Vereinigung ihrer verstorbenen Mitglieder, den Kollegen Georg Brunner, Boris Meissner und Heribert Zitzelsberger. 29 Mitglieder wurden neu aufgenommen, so dass die Vereinigung nun mehr als 570 Mitglieder zählt. In diesem Zusammenhang mag erwähnt werden, dass Herr Schulze-Fielitz auf der Mitgliederversammlung ausführlich über den Stand und die Zukunftsaussichten des wissenschaftlichen Nachwuchses berichtete.

Was den wissenschaftlichen Teil der Jahrestagung angeht, so diente er vor allem der Selbstvergewisserung über Stand, Methoden und Grenzen unserer Disziplin. Frau Juliane Kokott und Herr Thomas Vesting gingen den Konsequenzen von Europäisierung und Internationalisierung nach und untersuchten auf diese Weise die Konsequenzen der Veränderung ihres Gegenstandes für die Staatsrechtslehre. Die Kollegen Winfried Brugger und Christoph Gusy beschäftigten sich mit „Gewährleistung von Freiheit und Sicherheit im Lichte unterschiedlicher Staats- und Verfassungsverständnisse" und leisteten so auch einen Beitrag zur Nachbereitung der Ereignisse des 11. September in den USA. Was Stand und Methoden des Verwaltungsrechts angeht, so untersuchten Arno Scherzberg und Oliver Lepsius die Leistungsfähigkeit des Verwaltungsrechts bei der rechtlichen Risikosteuerung, und zwar im Hinblick auf die „Ermöglichung oder Begrenzung von Innovationen?“ Die Kollegen Rolf Gröschner und Johannes Masing zeichneten erste Konturen eines Informationsverwaltungsrechts.

Was den gesellschaftlichen Teil unserer Tagung angeht, so ist für den Empfang des ersten Abends eine Hamburger Besonderheit zu berichten; 
da es in Hamburg zwei Ausbildungsstätten für den juristischen Nachwuchs gibt, genoss die Vereinigung nicht nur die Gastfreundschaft der Universität Hamburg und ihrer juristischen Fakultät, sondern auch die der Bucerius Law School, die auch Gastgeberin des „Gesprächskreises Verwaltung" am Mittwochvormittag war, während der Arbeitskreis „Europäisches Verfassungsrecht“ in den Räumen der Universität tagte. Am Donnerstagabend war die Vereinigung Gast des Senates der Freien und Hansestadt Hamburg und wurde durch ihren Ersten Bürgermeister, Herrn Ole von Beust begrüßt. Der Freitagabend im Hotel „Hafen Hamburg" bescherte den Mitgliedern der Vereinigung nicht nur einen faszinierenden Blick auf den nächtlich beleuchteten Hamburger Hafen, sondern auch schmissige Songs der „Tailed Comedians“.

Der Sonnabend - traditionell der „Ausflugstag“ der Vereinigung führte uns in die traditionsreiche Hansestadt Lübeck. Nach einem Empfang im Rathaus und einer kundigen Stadtführung fand die diesjährige Jahrestagung mit einem gemeinsamen Mittagessen im „Haus der Schiffergesellschaft" ihren würdigen Abschluss.

G. F. S. 